\title{
DYNAMIC PERFORMANCE ANALYSIS AND CONTROL OF A SWITCHED RELUCTANCE MOTOR FOR ELECTRIC VEHICLE APPLICATIONS
}

\author{
A. A. Abd El-Salam \\ Mohamed A. El-khazendar \\ Electrical Power and Machines Engineering Department, \\ Faculty of Engineering, Tanta University, Egypt
}

\begin{abstract}
The driving motor of an electric vehicle should satisfy its specific requirements of both starting and providing an extended speed range at constant power within operating constraints (e.g. acceleration and gradeability). This paper investigates the capability of the switched reluctance motor (SRM) for electric vehicle (EV) applications by introducing a suitable controller. The proposed controller uses a fixed as well as an adapted switching angles control schemes to maximize the machine overload capability. Both Turn-on angle and conduction angle have great influence on developed torque and they can be chosen to maximize the operating speed range and hence extends the constant power region essentially required for EV. A nonlinear analysis for an $8 / 6 \mathrm{SRM}$ is developed and implemented in a simulation program based on Matlab/Simulink software package to investigate the motor dynamics. An experimentally measured flux linkagecurrent and static torque arrays are used to represent the machine data for simulation. The performance of SRM drive system with the proposed controller is introduced to study the effect of the proposed control scheme on current, torque, speed response and also constant power region. Motor currents and developed torques are obtained at different operating conditions and useful conclusions are obtained.

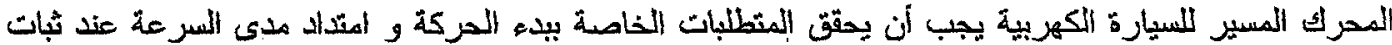

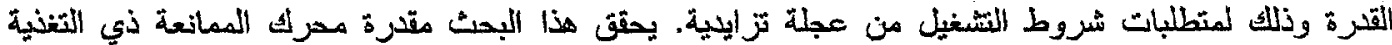

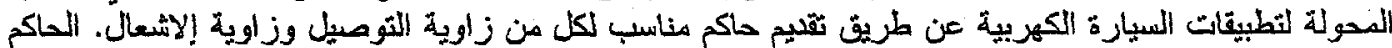

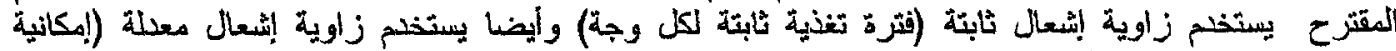

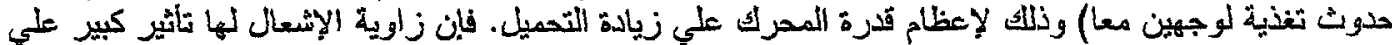

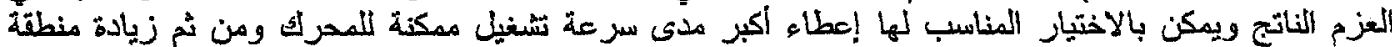

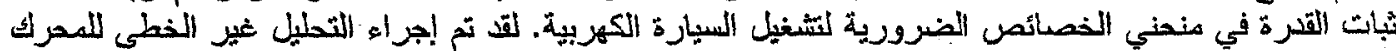

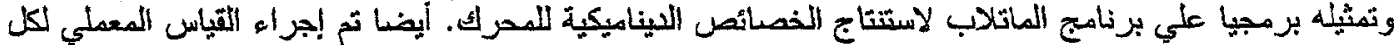

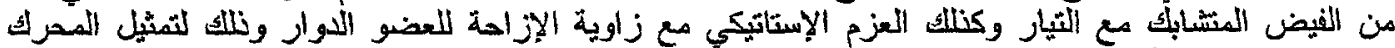

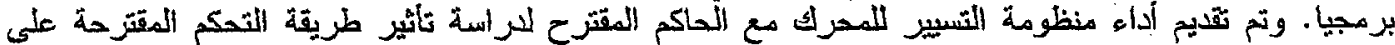

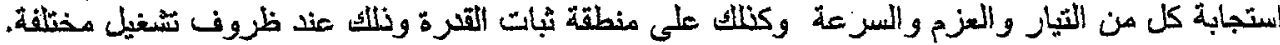

Keywords:

\section{INTRODUCTION}

Switched reluctance motor (SRM) has been emerging as an attractive solution for variable- speed drives and electric vehicles application due to several advantage such as robust structure and simplicity of its power converter. It has capability of high-speed operation with low cost and high efficiency. There are, however, several disadvantages of this machine. The disadvantages include:
1) acoustic noise generation;

2) torque ripple;

3) special converter topology requiring discrete insulated gate bipolar transistor (IGBT) modules;

4) excessive bus current ripple; and

5) The doubly salient structure combined with magnetic saturation nonlinearity is the main difficulty of this machine [2-4]. 
All of the above advantages as well as the disadvantages are quite critical for vehicle applications. Acceptable solutions to the above disadvantages are needed to get a viable drive for vehicle propulsion based on the SR machine. The developed torque is a function of both phase current and rotor position. It is also affected by saturation of partially overlapping stator and rotor poles [5]. To predict motor performance it is always necessary to find out the solution of a set of nonlinear differential equations representing the machine and it's power converter. Many trials had adopted the numerical finite element integration method to determine the static phase flux linkage and torque. Alternatively, different analytically methods are adopted [2-4]. The main problem of the motor performance analysis and control is the nature of relationship between the flux linkage and the current of each phase at different rotor displacement angles. A different control strategy has been applied to SRM to perform certain objectives such as smooth torque, and optimal commutation etc [5-8]. Comparative study of PI (Proportional, and Integral), sliding mode and fuzzy logic control for four-quadrant operation of SRM has suggested that the PI controller offers a simple control structure to achieve an optimum performance [6]. Hence, in this study, PI speed controller has been used in speed loop for predicting the drive performance.

Automotive market is one of the most successful applications of SRM technology [1]. It is expected that the use of SRM's and drives to grow in transportation applications where its high performance and low cost dictates its use. Electric vehicle performances require a high constant power to constant torque ratio. SRM, if designed and controlled appropriately, is capable of producing an extended constant power range.

Prototypes of SR machines have been designed, built, and tested for vehicle propulsion [12]-[15]. Most of these efforts concentrated on the machine design with emphasis given to design a high-density and a high-efficiency machine. Unfortunately, not much work has been done to develop a comprehensive controller for EV propulsion addressing all the relevant issues[9]. Most recently, an optimized controller for EV propulsion has been presented [16]. Also, a high performance fully digital SRM current controller for EV propulsion has been designed [9].

The available SRM is a $1 \mathrm{HP}$, four-phase, 8-6 pole configuration that has not been designed specifically for vehicle propulsion. However, it will be simulated and controlled with the adapted control parameters to be suited for $\mathrm{EV}$ applications. The predicted performances of the used SRM are obtained from the static measured data. These data are reused on dynamic simulation by using look-up table technique.

This paper presents a comprehensive controller for $\mathrm{EV}$ drives. An attempt has been made through machine control to maximize the SR performance, while paying particular attention to overcoming the difficulties. The controller is designed, based on the machine's measured characteristics, to maximize peak overload capability to be suited for EV. Lookup tables are used in the controller and interpolations are performed online between torque command, and speed to drive the motor at the optimal control parameters.

The proposed controller analyzes the performance of SRM drive with different values of fixed turn-on angles and on-line adapted turn-on angle. The first is used for a fixed conduction period with just one phase excitation. The second is used for overlapping the phase excitation that gives an acceptable performance of the drive. A comparative study between two cases is introduced. The turn -off angle remains fixed throughout the study. The drive performance in terms of speed, winding current and electromagnetic torque is presented. Simulated results pertaining to starting time, overshoot, steady state error, settling time, speed ripple and torque ripple are also presented.

\section{SWITCHED RELUCTANCE MOTOR DRIVE}

The standard drive configuration, (The $H$-bridge asymmetric type converter), is used to drive an $8 / 6$ four phase SR machine. It consists of one leg per phase. Each phase has two power switches and two diodes. The operation of this driver is shown in Fig. 1. * When both the switches are $O N$, then the winding is in energizing mode ( $+V$ is applied to phase winding).

* When both the switches are OFF, then the winding is in de-energizing mode ( $\mathrm{V}$ is applied to phase winding through two diodes).

* When any one of the switches is ON and another switch is OFF, then the winding is in current regulation mode.

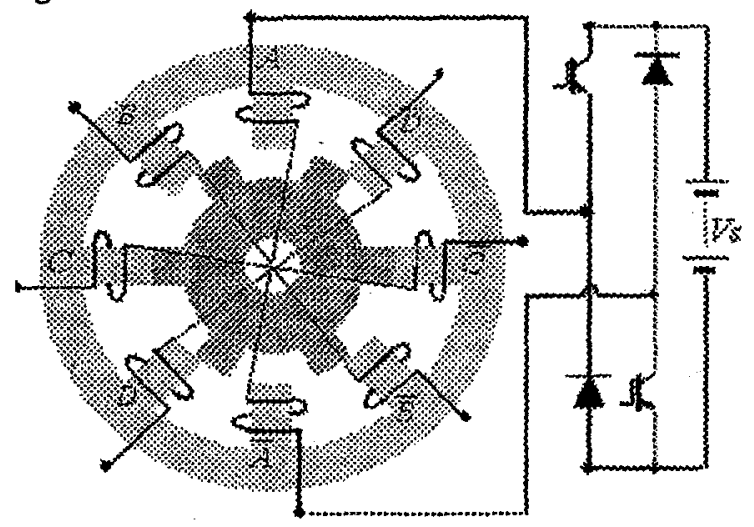

Fig. 1 Standard SRM per phase power converter 


\section{MODELING AND SIMULATION OF SRM}

\subsection{SRM Dynamics}

The operation and modeling of SRMs is entirely different from conventional motors. The complication in modeling is due to the highly nonlinear nature of the motor. There have been many attempts to model SRMs, which differ in variables chosen for solution, therefore requiring different data sets. It is convenient to choose the governing equations as follows.

Electromagnetic equation:

$V(\theta)=R i+\frac{d \psi(\theta, i)}{d t}$

where $\psi$ is the flux linkage in the phase winding, $V$ is the terminal voltage, $i$ and $R$ are phase current and phase winding resistance, respectively.

Mechanical equation:

$$
\begin{aligned}
& J \frac{d \omega}{d t}=T-T_{1}-K \omega \\
& \frac{d \theta}{d t}=\omega
\end{aligned}
$$

where $T$ is the electromagnetic torque, $T_{I}$ is the load torque, $K$ is the friction-damping coefficient, $\mathrm{J}$ is the moment of inertia of the rotor and $\omega$ is the rotor speed [7]. It is obvious that the above equations have no analytical solution as the flux linkage is a nonlinear function of current and rotor angle, hence numerical analysis is necessary.

\subsection{Simulation of SRM}

Instead of using a linear model of the SRM, predicting the dynamic performance of its non-linear characteristics is presented. Static experiments are performed to get the machine's data. This data have been saved in two look-up tables which are used for simulating this machine. One is the flux linkage vs. rotor position and phase current; and the other is the phase current and rotor position vs. torque. The simulation model has been made on matlab/simulink software. The data for the SRM can be obtained from Finite Element Analysis (FEM) calculations (if the motor is in the design stage) [8]. For an existing prototype in this paper, the data is measured from experimental tests. Flux linkage-current data $(\psi, i)$ at both aligned and an unaligned position are obtained by measurement. With the rotor locked at an aligned position, a variable ac voltage is applied to one phase winding and the current is recorded for each applied voltage. The phase impedance is calculated for each, then the phase inductance, and hence the flux linkage, is determined. The same procedure is repeated with the rotor held at an unaligned position. Once the aligned and unaligned flux linkage-current data is obtained, a family of these curves can be obtained for intermediate rotor positions to obtain, finally, the input table $\psi(\theta, i)$. The inductance profile $L(\theta, i)$ obtained from the static measurements is displayed in Fig. 2 for different values of phase current and rotor position. Figure. 3 shows the flux linkage vs. phase current and rotor position. The static torque vs. rotor position and phase current look-up table can also be obtained from an experimental test as shown in Fig.4. Figure 5 shows the simulated transient results of phase voltage, phase current, phase torque, speed and total torque at low and high speeds. Simulation at low-speed has been performed with a hysteresis current controller to limit the current level and then the torque as in Fig. 5(a). Single pulse control has been used for high-speed operation. The current shape is formed by the effect of back EMF at high speed as shown in Fig 5(b). Finally, this simulation has been done at turn-on angle $\theta_{o n}=0^{\circ}$ and turn-off at $\theta_{\text {off }}=25^{\circ}$.

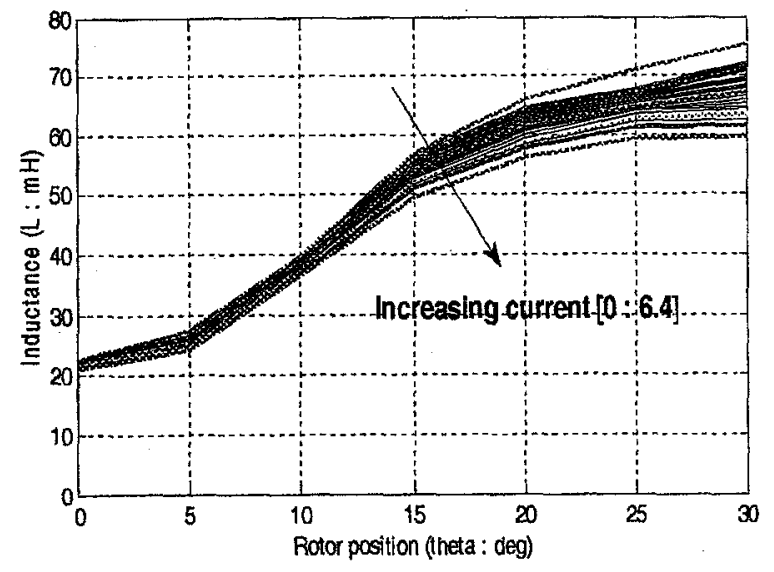

Fig. 2 Inductance profile $L(\theta, i)$

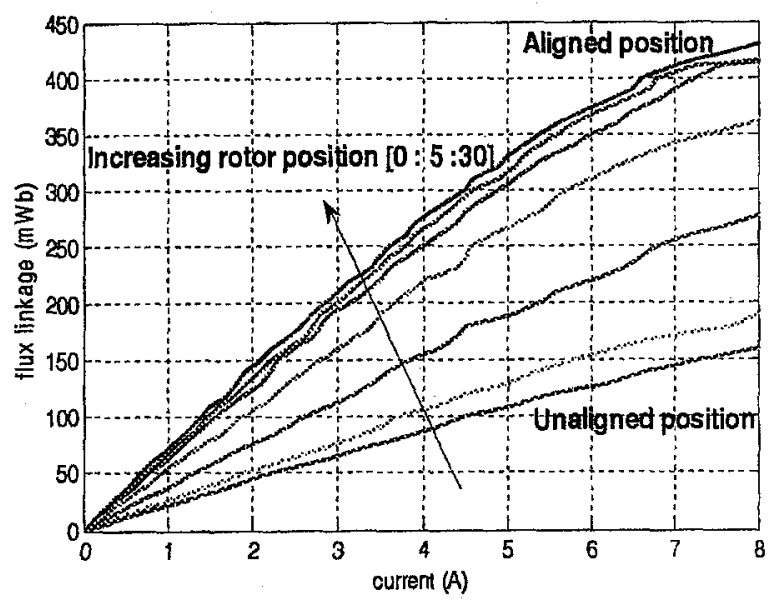

Fig. 3 Magnetic flux linkage $\psi(\theta, i)$ 


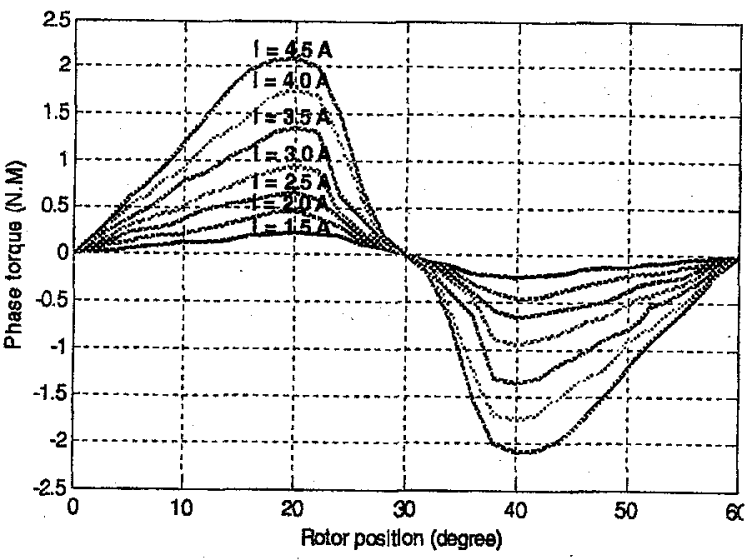

Fig. 4 Static performance, SR motor phase torque versus angular rotor position for various current levels
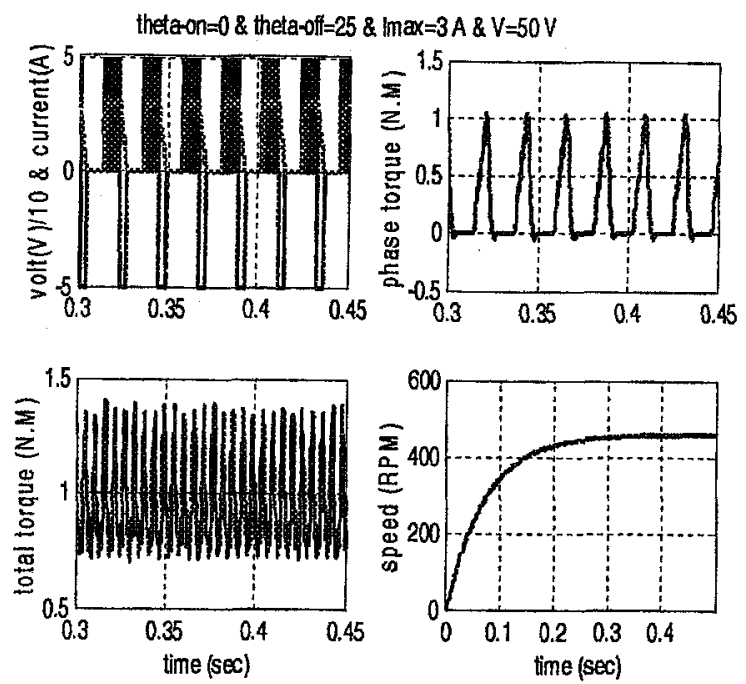

Fig. 5(a) Non-linear model and hysteresis current control at $\theta_{\text {on }}=0^{\circ}$, and $\theta_{\text {off }}=25^{\circ}$
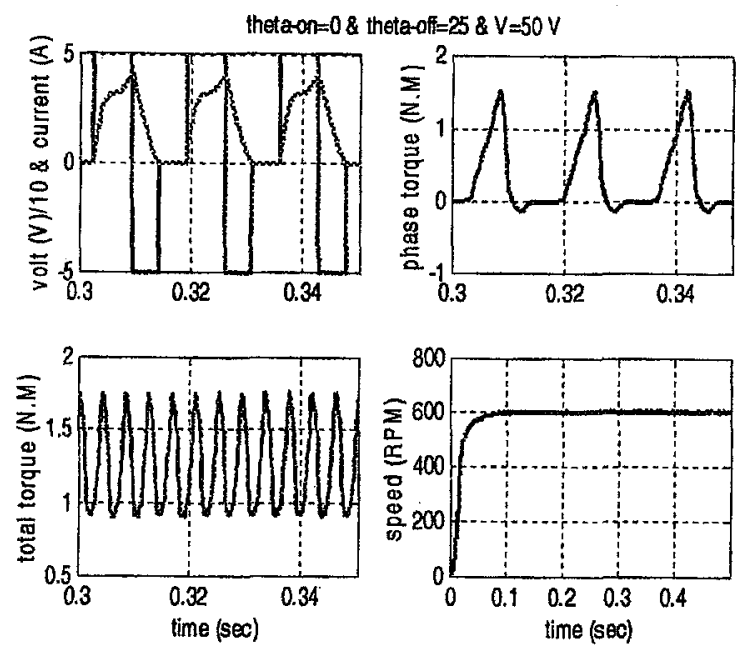

Fig. 5(b) Non-linear model and voltage control at $\theta_{\text {on }}=0^{\circ}$, and $\theta_{\text {off }}=25^{\circ}$, phase voltage, current, torque, total torque, and motor speed

\section{PREDICTING THE CONTROL \\ PARAMETERS OF SRM \\ 4.1 Predicting the Turn-on Angle}

Below base speed, the torque is limited by the imposed current limit ( $4 \mathrm{~A}$ ). Above this speed, the torque is limited by the generated voltage, which limits the build up current. After the steady state performance simulation of 8/6 SRM was studied, the predicted torque/speed characteristics at different turn-on angles would be obtained as shown in Fig. 6 . Turn-on angle has been changed by negative unit step (from $10^{\circ}$ to $0^{\circ}$ ). This helps to predict the turnon angle at any required average torque and speed by numerical inversion of the previous relation. This prediction data would be used to control the SRM.

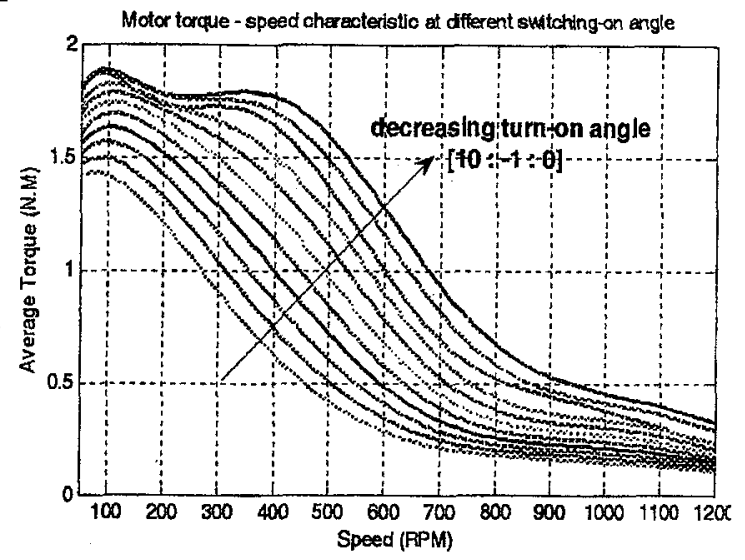

Fig. 6 Predicted average motor torque vs. speed at different values turn-on angle

4.2 Predicting the Current Command From the Torque. (Torque to current transformation).

In low-performance controllers, there is no special torque controller. In that case, the output of the speed controller can be treated as the torque command signal. Torque speed relation has been related at different current levels from steady state operation. These relations data has been stored on look-up table and then, have been used on torque command to current command transformation process. The predicted data are shown in Fig. 7.

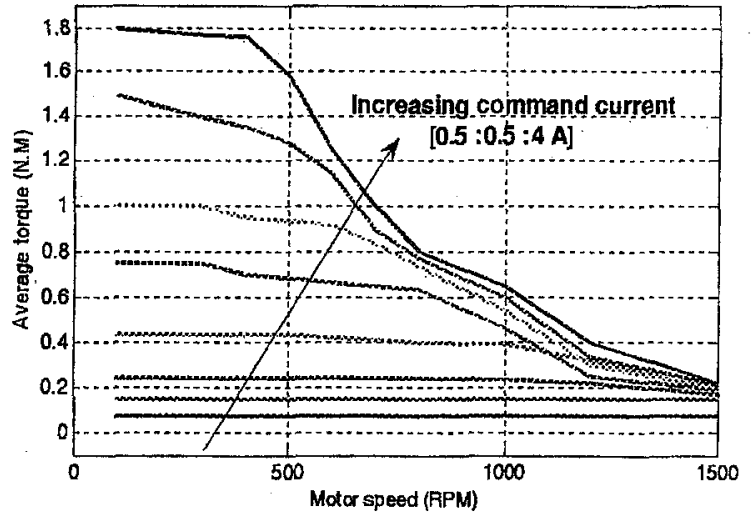

Fig. 7 Predicted average motor torque vs. motor speed at different values of reference current 


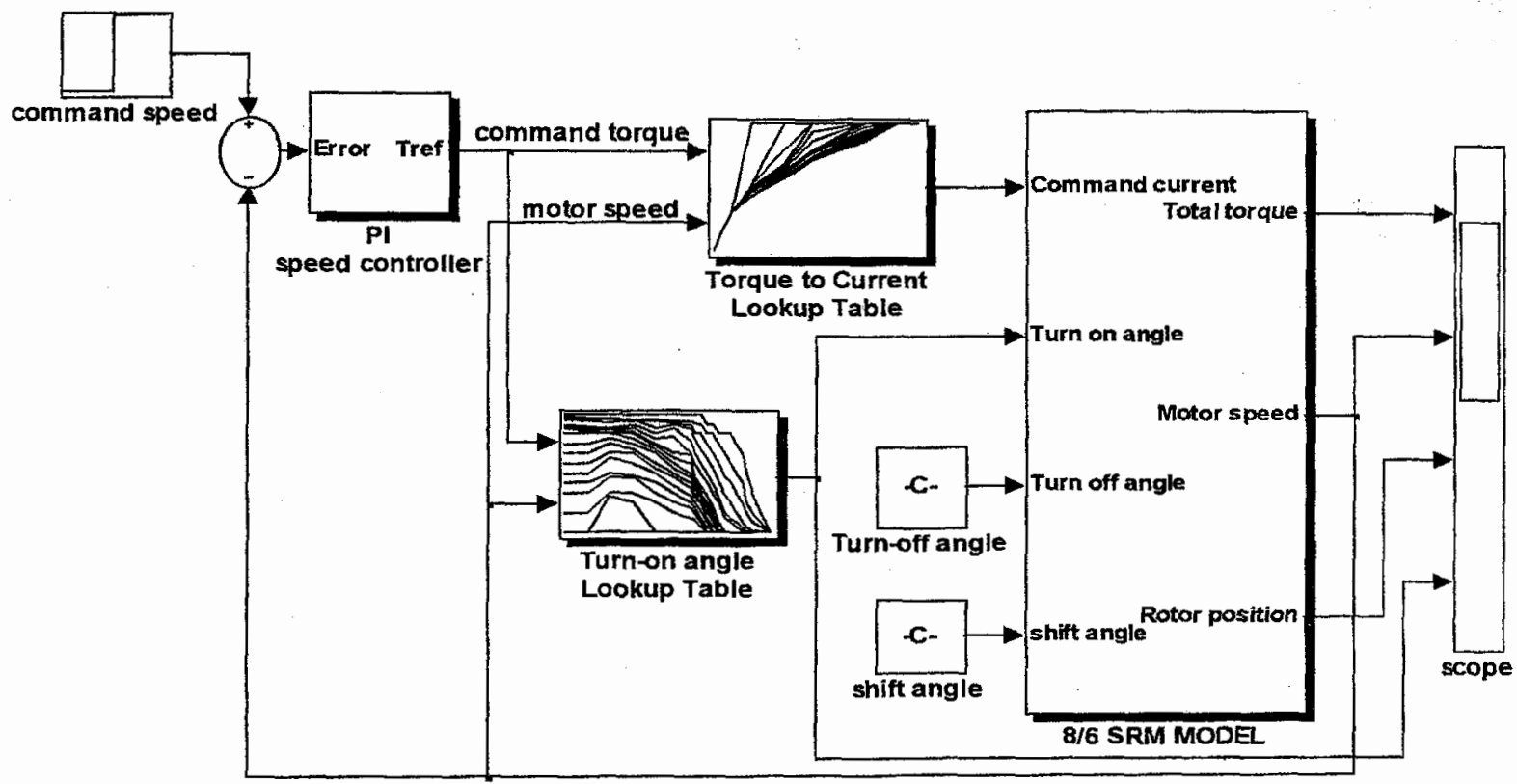

Fig. 8 Simulation block diagram of the 8/6 SRM and Control System

\section{CONTROL SYSTEM DRIVEN WITH PI SPEED CONTROLLER}

Figure 8 shows the simulation block diagram of the control system. The look-up tables, used in this control algorithm, are shown. An SR machine model is developed for this purpose. The rotor position is sensed by position sensor, the derivative of which gives the rotor speed $(\omega)$. The rotor speed is compared with set reference speed $\left(\omega^{*}\right)$ and the speed error $\left(\omega_{e}\right)$ is processed in PI speed controller. The speed error is given by:

$$
\omega_{e}=\left(\omega^{*}-\omega\right)
$$

The output of the speed controller is given by:

$T^{*}=K_{p}\left(\omega^{*}-\omega\right)+K_{i} \int\left(\omega^{*}-\omega\right) d t$

where, $T^{*}$ is the command torque, $K_{p}$ and $K_{i}$ are proportional and integral gain constants, respectively, of the speed controller and $\omega_{\mathrm{e}}$ is the speed error. The values of $K_{p}$ and $K_{i}$ depend on the parameters of the drive system. The controller gains are selected by comparing the effect of $K_{p}$ and $K_{i}$ on the speed response of the drive [1]. The simulation is performed at $\left(\mathrm{K}_{\mathrm{i}}=2 \& \mathrm{~K}_{\mathrm{p}}=0.25\right)$.

\section{SWITCHING ANGLE CONTROL.}

Maximization of the developed torque is an important issue for SRM to be made suitable for EV applications. In order to assure a maximum torque zone and thus a maximum average torque in the motoring mode of SRM, the best turn-on angle has to be chosen. The extreme limits of the turn-on angle are established geometrically. As a function of the specific application, these limits can vary with the proposed strategy. For the first phase of the motor, the minimum turn-on angle is represented by the rotor position corresponding to the unaligned rotor position $\left(\theta_{\mathrm{on}}=0^{\circ}\right)$ and the maximum is $\left(\theta_{\mathrm{on}}=10^{\circ}\right)$ when the turn-off angle is fixed at $\left(\theta_{\text {off }}=25^{\circ}\right)$. The procedure consists of finding the advance turn-on angle for a specific reference current (or torque) and rotor speed. Therefore, the control scheme and torque-speed characteristics have been processed and investigated with two control parameters. These control parameters are turn-on angle and current level.

6.1 Control with only one phase excitation.

Simulation responses under constant PI speed controller gains and constant switching angles (turnon and turn-off angle) were performed. The conduction angle is fixed at the step angle $\theta_{s}$ $\theta_{s}=360 *\left(\frac{1}{N_{r}}-\frac{1}{N_{s}}\right)=15^{\circ}$

Phase excitation sequence is then A-B-C-D without any overlapping for clockwise rotation. Figure 9 shows the simulation response to different command speeds at $\left(\theta_{o n}=1\right.$ and $\left.\theta_{\text {off }}=16\right)$. A good response has been obtained at low speed. Overshoot at medium and high speeds were viewed and relatively large a time is required to reach steady state as shown in Fig 9(a). This overshoot can be decreased by adjusting the value of PI controller gains. In addition, at low speed, the current was chopped at low command level (1A). At medium speeds, the current 
was chopped at higher current level (3A) to increase the developed torque. At high speeds, the current is limited by back EMF at rated value ( $4 \mathrm{~A})$, and there is no current chopping as shown in Fig. 9(b and c). The maximum speed is limited at low speed (450 RPM), corresponding to the torque production period. Figure 9(d) shows the total instantaneous developed torque vs. motor speed. It is obvious that the maximum or average developed torque is constant, except for the higher speeds, at which the constant power region is very small.

\subsection{Control with step variation of turn-on angle.}

Overlap of the motor phase's results in increasing the conduction period. This leads to an increase in the produced torque and then the motor speed. The conduction period can be determined either by fixing the turn-on angle at $\left(\theta_{o n}=0^{\circ}\right)$ and delaying the turnoff angle ( $\theta_{\text {off }}=15^{\circ}: 25^{\circ}$ ) or by fixing the turn-off angle at $\left(\theta_{f f}=25^{\circ}\right)$, as maximum value, and advancing the turn-on angle $\left(\theta_{\text {on }}=10^{\circ}: 0^{\circ}\right)$. The later technique has been suggested. Figure 10 shows the response at $\left(\theta_{o n}=10^{\circ}, 8.5^{\circ}\right.$, and $\left.0^{\circ}\right)$. The turn-off, angle was fixed at $\left(\theta_{\text {off }}=25^{\circ}\right)$. At low speeds, the current was chopped at low command level (1A) with fixed turn-on angle $\left(\theta_{\text {on }}=10^{\circ}\right)$. High performance was obtained at low speeds. At medium speed, the current was chopped at higher level (2.3A). Therefore, the total developed torque was increased. At high speeds, the current is limited by back EMF at rated value (4A), and there is no chopping. The maximum speed (360 RPM) is limited corresponding to the torque production period (region 1). Advancing turn-on angle increases the conduction period and then torque production period. Thereafter, the speed increases to follow the reference speed (region 2 and region 3 ). This is shown in Fig. 10 ( $a, b$, and $c)$.

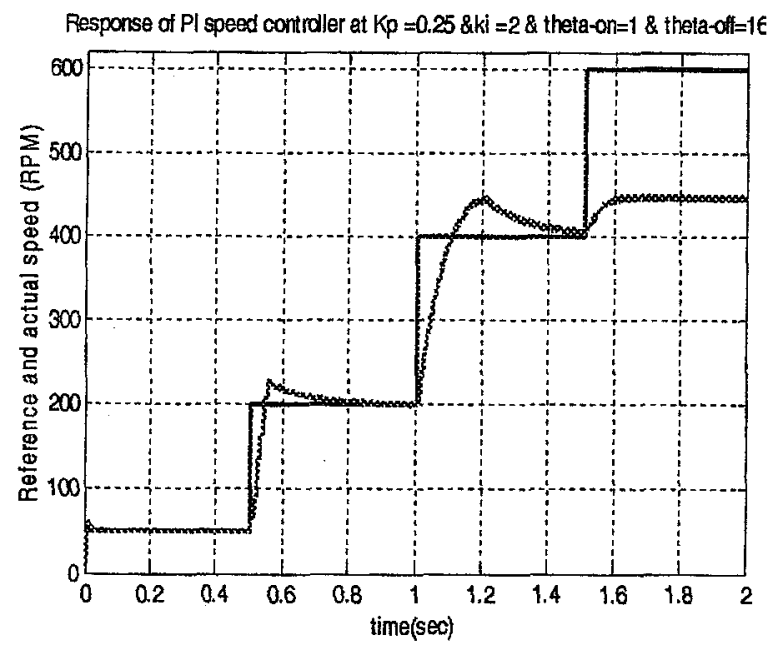

Fig. 9 (a)
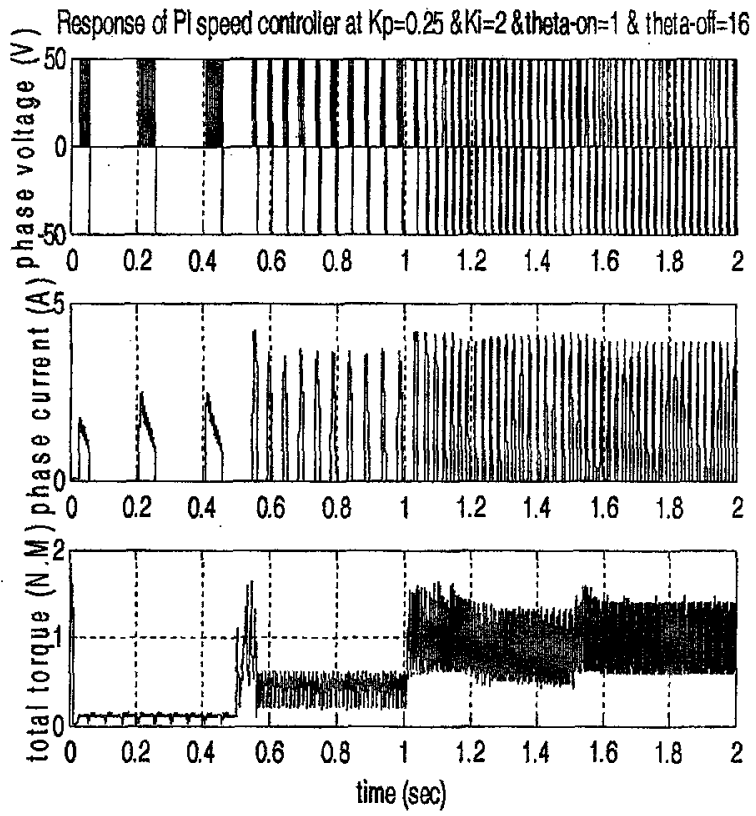

Fig. 9 (b)
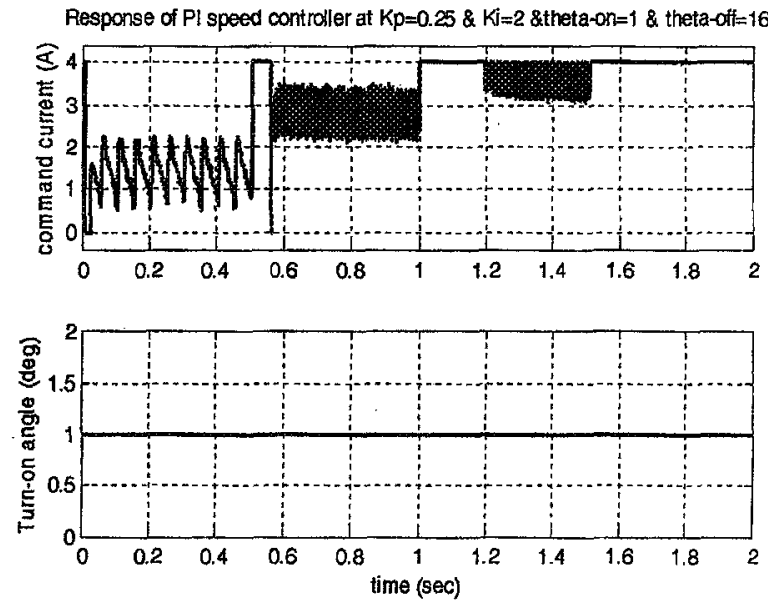

Fig. 9 (c)

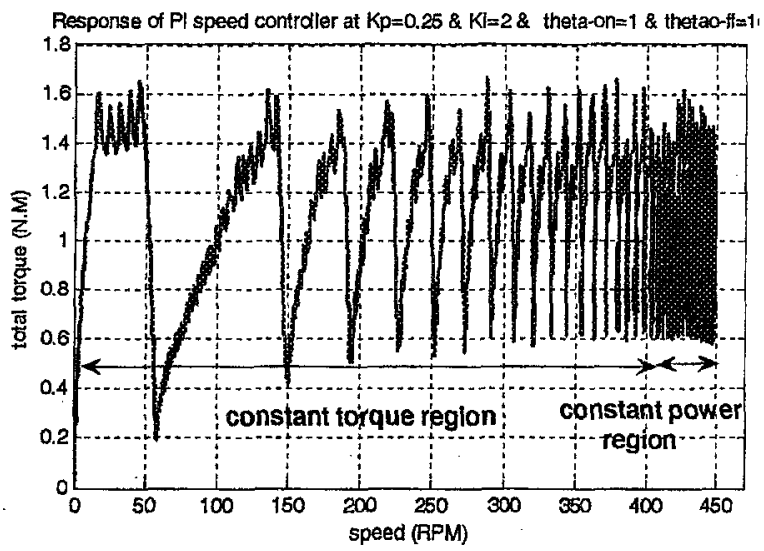

Fig. 9 (d)

Fig. 9 Control with only one phase excitation 


\subsection{Control with on-line adaptation of the turn-on angle (proposed method).}

The phase current is a function not only of command current, but also, of the turn-on angle. This is why at low speeds; the phase current is chopped at low level while the command current at high level with low turn-on angle. At low speeds, the system response was overshoot and oscillated. At medium speeds, the speed response has been improved.

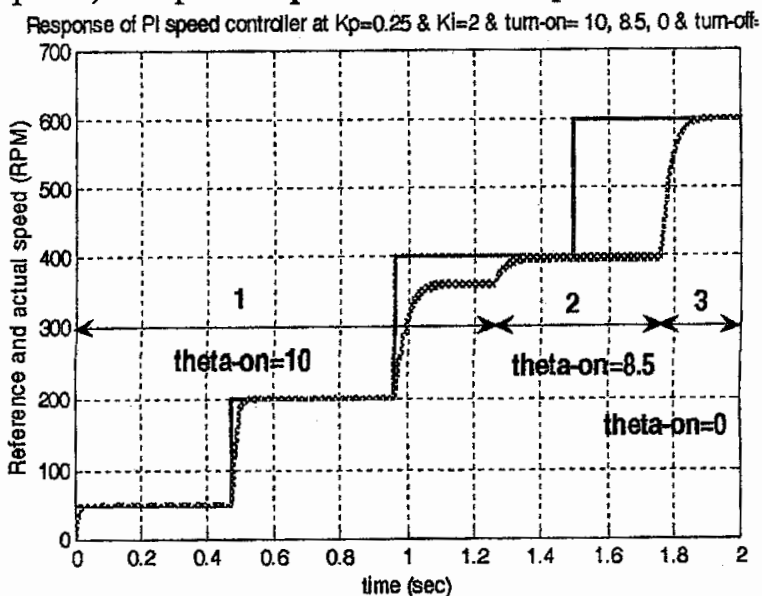

Fig. 10 (a)

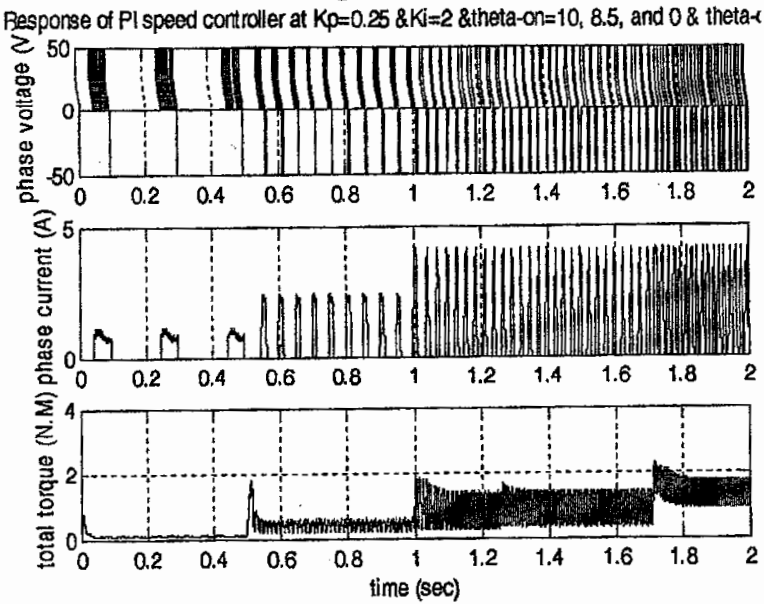

Fig. 10 (b)

Response of Plspeed controller at $K p=0.25$ \& Kl=2 \&theta- $-0 n=10,8.5$, and 0 \& theta-0
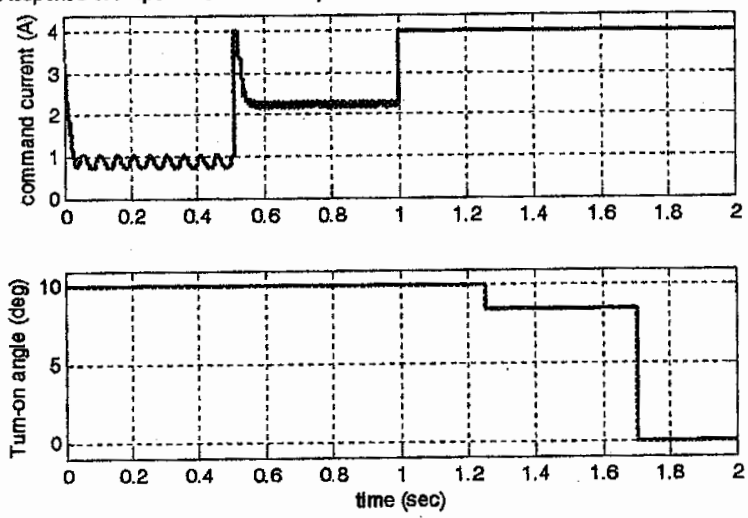

Fig. 10 (c)

Fig. 10 Control with step variation of turn-on angle
The current has been chopped at the command current with adapted turn-on angle at advanced position. At high speeds, a highly controller response was obtained with a highly speed response. The current is limited at rated value by back EMF without any chopping. The turn-on angle is advanced at the minimum reluctance position $\left(\theta_{o n}=0^{\circ}\right)$. All these cases are shown in Fig. $11(a, b$, and c). Figure $11(d)$ shows the effect of proposed controller to the torquespeed curve. It is obvious that the constant power region has been extended. Also, the maximum or average torque is constant at a higher value. These results are related to the available motor. Therefore, this extension can be more increased by another specially designed motor with the same proposed controller scheme.

The speed controller gains have been adjusted according to the drive's speed as shown in Fig. 12 (a, $b$, and c) with $K_{p}=100$ and $K_{i}=5$. It is obvious that the system response with the proposed controller has been improved.

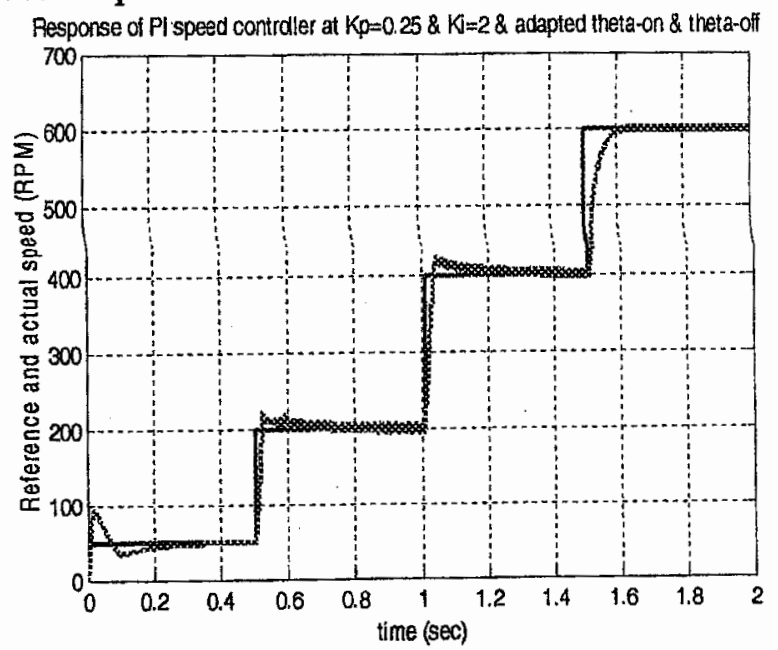

Fig. 11 (a)

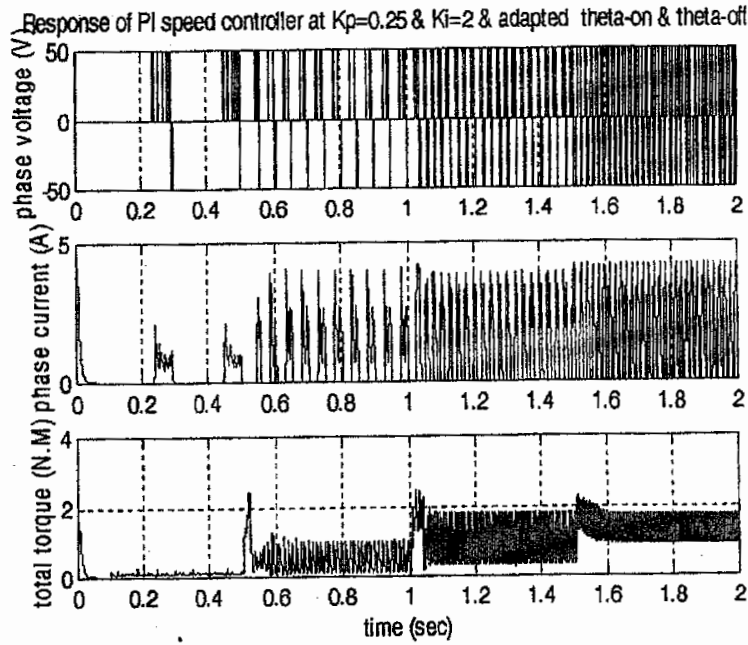

Fig. 11 (b) 

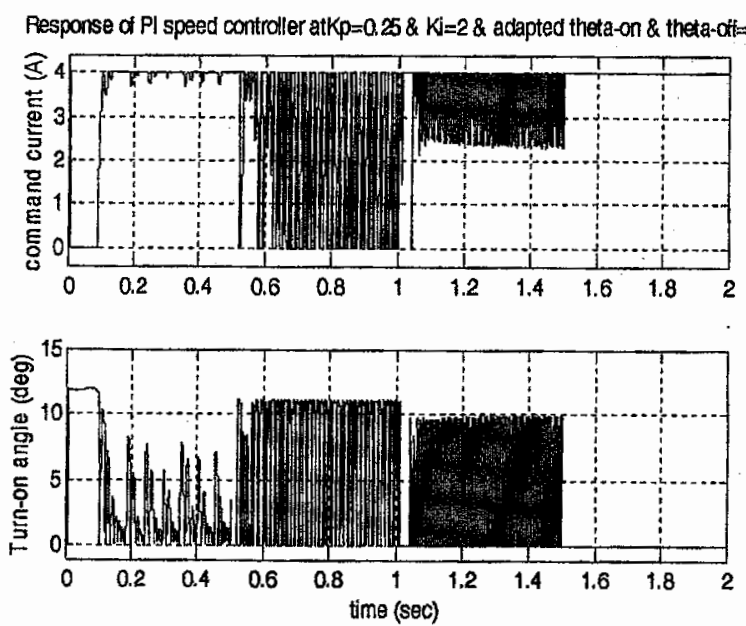

Fig. 11 (c)

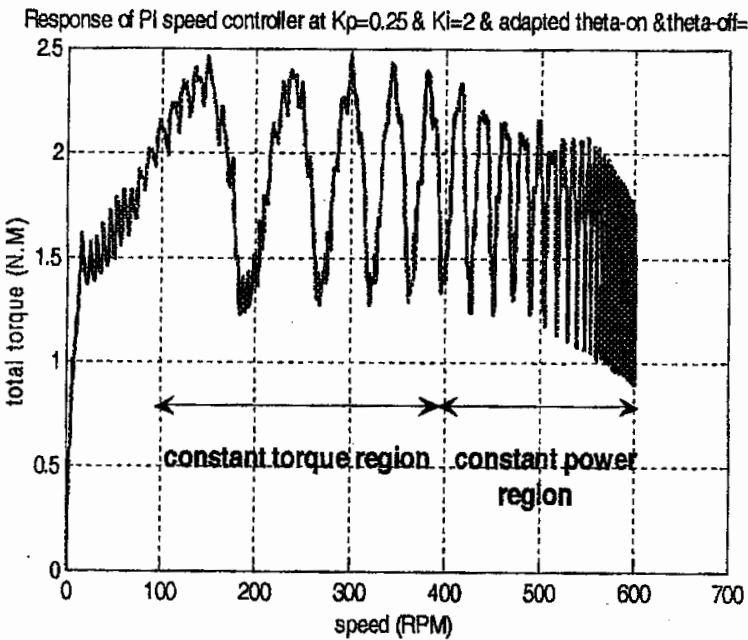

Fig. 11 (d)

Fig. 11 Control with on-line adaptation of the turn-on angle (proposed method)

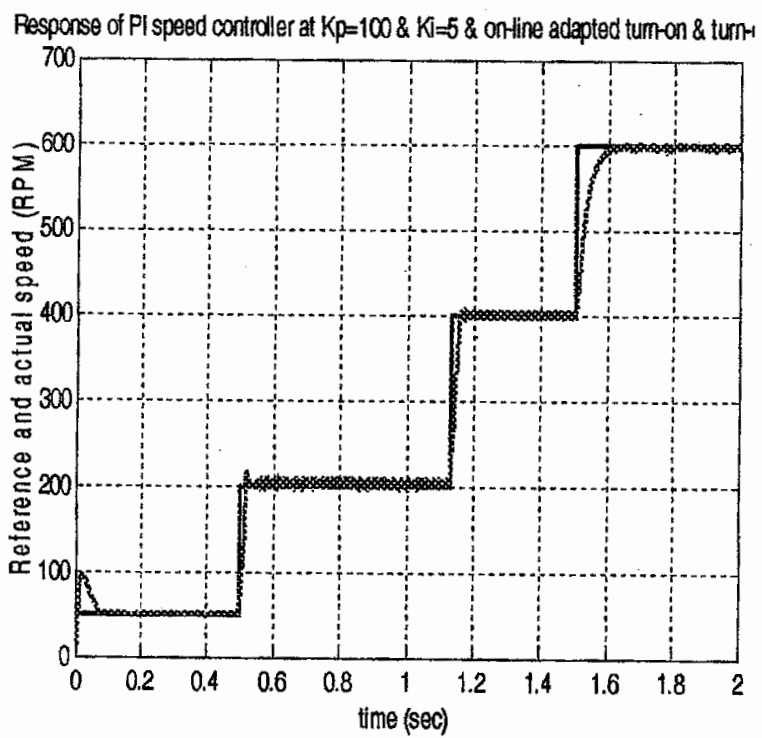

Fig. 12 (a)
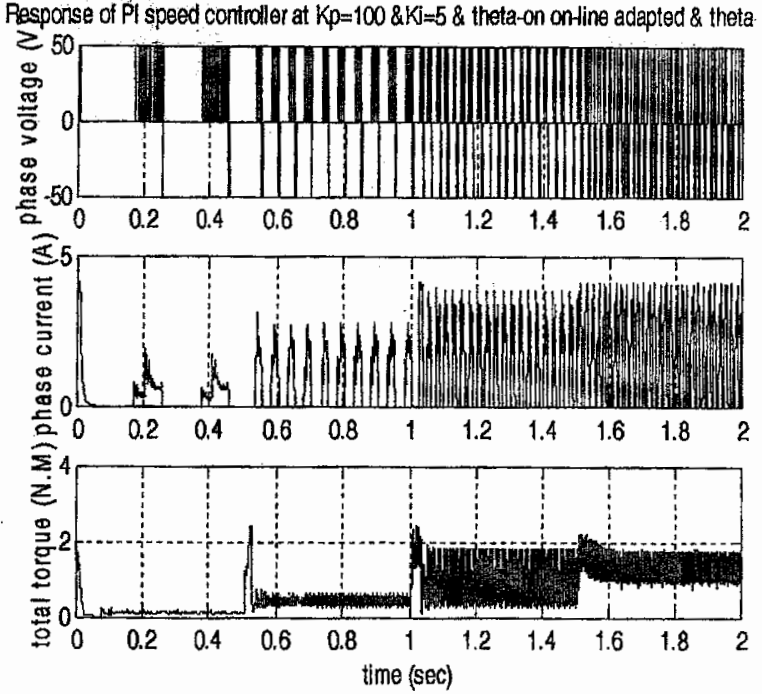

Fig. 12 (b)

on-ine adaptation of control parameters to PI speed controller with constant gair
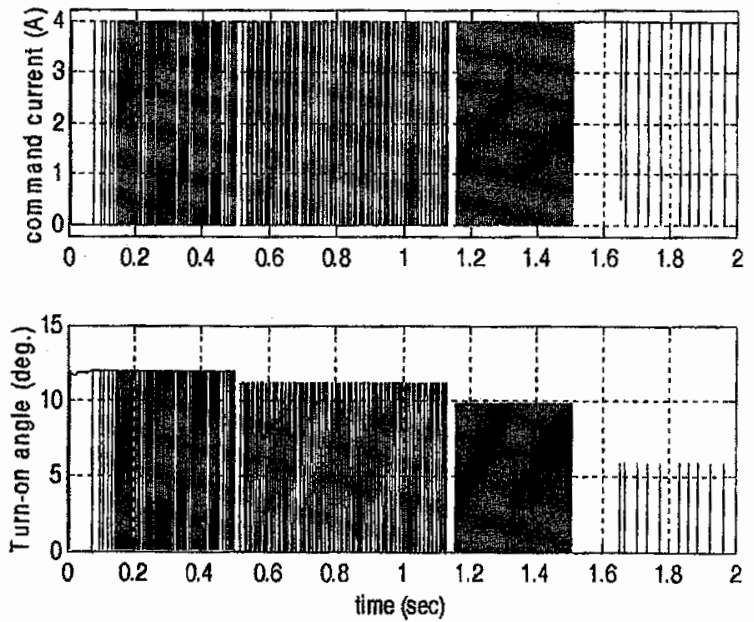

Fig. 12 (c)

Fig. 12 Effect of PI speed controller gains to system performances

\section{CONCLUSION}

A nonlinear simulation for an $8 / 6$ switched reluctance motor has been implemented to study its dynamics and control characteristics under various turn-on angles. Experimentally measured data have been used for simulations. A dynamic analysis has been introduced for torque, speed and angle control. Control parameters have been proposed and different excitation modes were given. It has been found that control with only one phase excitation restricts both of the developed torque and maximum speed which, in turn, restricts the constant power region. Accordingly, this method is not suitable for EV application. It has been also found that the use of the proposed controller by advancing the turn-on angle increases both the motor developed torque and its maximum speed. Hence, this control approach 
increases the maximum speed and then, the constant power region in torque/speed curves. Good performance has been obtained by the careful choice of PI speed controller gains. The obtained results suggests that SRM provided with suitable control can be used for electric vehicle propulsion.

\section{REFERENCES}

[1] J.M Stephenson and J.Corda "computation of torque current in double salient reluctance motors from non-linear magnetization data, "proc IEE, vol. 126, no.5, may, 1979, pp. 393396.

[2] J.Corda and J.M Stephenson "Analytical estimation of the minimum and maximum inductances of a double salient motor" proc of the international conference on stepping motors and systems, university of Leeds, UK, sept, 1979, pp.50-59.

[3] Shuyu cao, K.J teseng, "A new method for accurate analytical modeling of switched reluctance motor", PEDES'98 vol. II, December 1998, pp. 540-545.

[4] C. Rochford, R.C Kavanaph, M.G. Egan and J.M.D. Murphy, " switched reluctance motors using self-learning techniques", ENE'93, PP.1419.

[5] Krzysztof Russa, Iqbal Husain and Malik E. Elbuluk, "a self-Tunning controller for switched reluctance motors", IEEE-PE Tran, vol. 15, No. 3, May 2000.

[6] Catalin serban dragu and ronnie belmans katholieke "optimal firing angles control for four-quadrant operation of an $8 / 6 \mathrm{SRM}^{\prime \prime}$, EPE 2003 - Toulouse.

[7] F. Soares and P.J. Costa Branco, "Simulation of a 6/4 Switched Reluctance Motor Based on Matlab/Simulink Environment", 2001.

[8] F.Ismael, M.M.Khater, H.El kashab, and A. Oshiba " dynamic performance analysis and control of a switched reluctance motor 'MEPCON'2003, minoufiya university.

[9] P. J. Blake, R. M. Davis, W. F. Ray, N. N. Fulton, P. J. Lawrenson, and J. M. Stefenson, "The control of switched reluctance motors for battery electric road vehicles," in Proc. Int. Conf. PEVD, May 1984, pp. 361-364.
[10] J. M. Miller, A. R. Gale, P. J. McCleer, F, Leonardi, and J. H. Lang, "Starter-alternator for hybrid electric vehicles: Comparison of induction and variable reluctance motors and drives," in Conf. Rec. IEEE-IAS Annu. Meeting, vol. 1, 1998, pp. 513-523.

[11] Khwaja M. Rahman, and Steven E. Schulz, "High-Performance Fully Digital Switched Reluctance Motor Controller for Vehicle Propulsion", IEEE Transations on industry applications, vol. 38, no. 4, july/august 2002

[12] J. Reinert, R. Inderka, M. Menne, and R. W. D. Doncker, "Optimizing performance in switched reluctance drives," IEEE Ind. Applicat. Mag., vol. 6, pp. 63-70, July/Aug. 2000.

\section{APPENDIX}

Motor and controller specifications:

- $\mathrm{N}_{\mathrm{s}}=8$ : Number of stator poles.

- $N_{\mathrm{r}}=6:$ Number of rotor poles.

- $m=4$ : Number of phases.

- Bore diameter or rotor pole tips $=75.508 \mathrm{~mm}$.

- Air gap length $=0.3 \mathrm{~mm}$.

- Core axial length $=100 \mathrm{~mm}$.

- Stator pole height $=22.24 \mathrm{~mm}$.

- Rotor pole height $=17.311 \mathrm{~mm}$.

- Outer diameter $=140 \mathrm{~mm}$.

- Shaft diameter $=18 \mathrm{~mm}$.

- $\beta_{s}=20^{\circ}$ : Stator pole breadth.

- $\beta_{r}=26^{\circ}$ : Rotor pole breadth.

- $\frac{360}{N_{s}}=45^{\circ}:$ Stator pole pitch.

- $\frac{360}{N_{r}}=60^{\circ}:$ Rotor pole pitch.

- Minimum inductance zone $=14^{\circ}$

- Rising and falling inductance zone $=20^{\circ}$

- Maximum inductance zone $=6^{\circ}$

- Number of turns per phase $=260$ turns.

- Conductor diameter $=0.8 \mathrm{~mm}^{2}$.

- Motor power (designed value) $=1$ H.P.

- Phase current amplitude $=4 \mathrm{~A}$.

- Used DC voltage $=50 \mathrm{~V}$.

- Turn-off angle $=25^{\circ}$.

- Inertia coefficient $=0.002 \mathrm{Kg} \cdot \mathrm{m}^{2}$

- Friction coefficient $=0.002 \mathrm{~N} . \mathrm{m} . \mathrm{s} / \mathrm{rad}$ 